\title{
Gamma Knife Radiosurgery for Large Vestibular Schwannomas: A Canadian Experience
}

\author{
F.A. Zeiler, M. Bigder, A. Kaufmann, P.J. McDonald, D. Fewer, J. Butler, \\ G. Schroeder, M. West
}

\begin{abstract}
Object: To review our institutional experience with Gamma Knife (GK) stereotactic radiosurgery in treating large vestibular schwannomas (VS) of 3 to $4 \mathrm{~cm}$ diameter. Methods: We conducted a retrospective cohort review of all patients treated with GK for VS at our institution between November 2003 and March 2012. Data on age, sex, VS volume, location and maximal diameter, House-Brackmann (HB) facial nerve scores pre and post-GK, Gardner-Robertson (GR) hearing score pre and post-GK, GK treatment parameters, VS response time, complications and clinical outcome was recorded Results: A total of 28 patients during the defined time period were identified. Three patients were lost to follow-up. Mean follow-up was 34.5 months. Tumor control occurred in $92 \%$, and was maintained in $85.7 \%$ at two years. Facial nerve or hearing preservation occurred in all treated compared to pre-GK status, as per HB and GR grading. Transient complications occurred in $80 \%$. Temporary vestibular dysfunction occurred in seven patients (28\%). One patient $(4 \%)$ had the permanent complication of worsening pre-GK hemifacial spasm. Four patients (16\%) developed hydrocephalus post-GK. Conclusion: GK stereotactic radiosurgery as a primary treatment modality for large VS can provide acceptable tumor control rates with good facial nerve and hearing preservation, and low complication rates.
\end{abstract}

RÉSUMÉ: Radiochirurgie par scalpel gamma dans les schwannomes vestibulaires de grande taille. Objectif : Le but de l'étude était de revoir notre expérience institutionnelle de l'utilisation de la radiochirurgie stétéotaxique par scalpel gamma (SG) dans le traitement des schwannomes vestibulaires (SV) de grande taille, soit de 3 à $4 \mathrm{~cm}$ de diamètre. Méthode : Nous avons effectué une revue rétrospective de cohorte de tous les patients traités par SG pour un SV dans notre institution entre novembre 2003 et mars 2012. Les données sur l'âge, le sexe, le volume du SV, sa localisation et son diamètre maximal, les scores à l'échelle de House-Backmann (HB) pour le nerf facial avant et après traitement par le SG, le score à l'échelle de Gardner-Robertson (GR) pour l'audition avant et après traitement par le SG, les paramètres de traitement par le SG, le temps de réponse du SV, les complications et l'issue clinique ont été colligés. Résultats : Nous avons identifié 28 patients qui ont été traités pendant cette période. Trois patients ont été perdus de vue. Le suivi moyen des patients était de 34,5 mois. Un contrôle de la tumeur a été constaté chez $92 \%$ des patients et il était maintenu après 2 ans de suivi chez $85,7 \%$ des patients. L'intégrité du nerf facial ou de l'audition était préservée chez les patients traités par rapport à leur état préSG, selon les échelles HB et GR. Des complications transitoires sont survenues chez $80 \%$ des patients. Une dysfonction vestibulaire temporaire a été observée chez 7 patients (28\%). Un patient (4\%) a présenté une complication permanente, soit une aggravation d'un spasme hémifacial préexistant. Quatre patients (16\%) ont présenté une hydrocéphalie postSG. Conclusion : La radiochirurgie stéréotaxique par le SG comme modalité de traitement initial pour un SV de grande taille offre des taux acceptables de contrôle de la tumeur avec une bonne préservation du nerf facial et de l'audition et un faible taux de complications.

Can J Neurol Sci. 2013; 40: 342-347

The treatment of small to medium sized vestibular schwannomas (VS) with Gamma Knife (GK) stereotactic radiosurgery is a well-documented treatment alternative to surgical resection, with prospective nonrandomized trials demonstrating facial nerve and hearing preservation rates favoring $\mathrm{GK}$ over microsurgery ${ }^{1,2}$. Tumor control rates have been described upwards of $94 \%$ in recent literature, with acceptable complication rates ${ }^{3}$ when compared to microsurgery. Long term actuarial resection-free control rates have been documented at $98.3 \% \%^{4-6}$, emphasizing the lasting effect post-GK.

Larger VS pose a difficult clinical challenge, with many authors favoring surgical resection due to concerns around radiation dosing and side effects. Differing recommendations currently exist in the literature in terms of the degree of resection. Recently, the suggestion of sub-total resection in order to reduce the volume to one more amenable to GK has arisen.
Using the combination of surgery and radiation, clinical and radiologic control has been documented at $92 \%$ and $90 \%$ respectively, with high facial nerve preservation ${ }^{7}$.

The role of stereotactic radiosurgery as a primary treatment modality in large VS (greater than $3 \mathrm{~cm}$ extra-cannicular diameter) has yet to be determined. Stereotactic radiosurgery

From the Section of Neurosurgery, Department of Surgery (FAZ, MB, AK, PJM, MW), Department of Radiation Oncology (JB, GS), University of Manitoba, Winnipeg, Manitoba, Canada.

Received October 24, 2012. Final Revisions Submitted November 22, 2012. Correspondence to: Frederick A Zeiler, Section of Neurosurgery, University of Manitoba, Health Sciences Center, GB-1 820 Sherbrook Street, Winnipeg, Manitoba R3A 1R9, Canada. Email: umzeiler@cc.umanitoba.ca. 
with GK for VS 3-4 cm maximal diameter as an isolated treatment, without surgical resection, has demonstrated an $89 \%$ tumor control rate at two years ${ }^{8}$ in the literature so far. Hearing, facial nerve, and trigeminal nerve preservation rates in these large VS treated with GK have been documented at 58\%, 91\%, and $86 \%$ respectively ${ }^{9}$.

Within this study we describe a retrospective cohort study of our institutional experience in treating large VS primarily with GK stereotactic radiosurgery between November 2003 and December 2011. The goal of the study is to define the tumor control rates, and outline the complication rates with emphasis on hearing and facial nerve preservation.

\section{MethodS}

We conducted a retrospective cohort study of all patients treated for large VS, size 3 to $4 \mathrm{~cm}$ in maximal diameter, at our institution up to and including March 2012. Utilizing a prospectively maintained database of all GK treatments at our institution, we obtained a list of all patients treated for VS of any size. There were 235 patients treated with GK for VS at the Winnipeg Centre for Gamma Knife Surgery in Winnipeg, Manitoba between November 2003 (the start of GK at our center) and March 2012. We subsequently checked each treatment plan manually, as recorded in our outpatient neurosurgery charts in order to screen out all of those patients with VS outside of 3 to $4 \mathrm{~cm}$ in maximal diameter. All data collection and chart review was conducted physically in the Section of Neurosurgery.

We recorded data on age, sex, VS volume, location and maximal diameter, House-Brackmann (HB) facial nerve scores pre and post-GK, Gardner-Robertson (GR) hearing score pre and post-GK, GK treatment parameters, VS response time, complications and clinical outcome. Post-radiosurgery assessments were conducted by the treating neurosurgeon involved with GK planning and treatment. Post-radiosurgery audiology assessment was typically conducted at yearly intervals, ordered by the patient's primary care physician.

Of note, GR grades were derived from the post-treatment audiology reports which were described as "unchanged" by both the audiologist and family doctor in letter form. Rarely did we have the objective audiology data available in our charts.

Post-treatment magnetic resonance imaging (MRI) interpretation was conducted by both neuro-radiology and the treating neurosurgeon. Maximum diameter was determined in the axial plane using caliper measurement on digital imaging. These measurements were compared to pre-radiosurgery MRI assessment.

Telephone follow-up occurred via GK nursing staff in between in-person surgeon appointments. This study was approved by the Health Research Ethics Board at the University of Manitoba.

\section{RESUlTS}

\section{Demographics and Presentation}

We defined large VS as those with an extra-cannicular maximal diameter greater than $3 \mathrm{~cm}$. Of the 235 patients treated during this time frame, 28 patients met the criteria for having large VS and were included in the review. Two (7.1\%) patients had neurofibromatosis type 2 (NF-2). Demographic and presenting tumor data can be seen in Table 1 .

There were 15 male $(54 \%)$ and 13 female (46\%) patients, with an average age of 56 years (range: 26-85). Twelve (42.3\%) patients had previous resection of their VS remote from their GK treatment. These 12 patients had received surgery as a primary treatment modality in the past, and not as a subtotal resection with the plan for future stereotactic radiosurgery.

Three of the 28 patients $(10.7 \%)$ treated were lost to followup. One of the patients had NF-2. Their clinical characteristics are displayed in Table 2. These patients were excluded from the

Table 1: Demographic data for all patients with follow-up

\begin{tabular}{|c|c|c|c|c|}
\hline Demographic Category & & $\begin{array}{c}\text { Patients with } \\
\text { Follow-Up }(n=25)\end{array}$ & $\begin{array}{l}\text { Two Year Follow-up } \\
\text { Group }(n=14)\end{array}$ & $\begin{array}{l}\text { Previous Surgical } \\
\text { Resection }(\mathrm{n}=12)\end{array}$ \\
\hline Age (years) & & 26-85 (avg: 56) & 32-85 (avg: 57) & 49-85 (avg: 63) \\
\hline \multirow[t]{2}{*}{ Sex } & Male & $13(52 \%)$ & $8(57.1 \%)$ & $7(58.3 \%)$ \\
\hline & Female & $12(48 \%)$ & $6(42.9 \%)$ & $5(41.6 \%)$ \\
\hline Average AN Diameter (cm) & & 3.28 & 3.31 & 3.21 \\
\hline Average $\%$ coverage & & 97.2 & 96.3 & $96.7 \%$ \\
\hline Average $50 \%$ isodose line (Gy) & & 12.5 & 12.5 & 12.5 \\
\hline $\begin{array}{l}\text { Average Time Until VS radiological } \\
\text { response (months) }\end{array}$ & & 20.4 & 24.8 & 19.5 \\
\hline Total Number with Tumor Control & & $23(92.0 \%)$ & $12(85.7 \%)$ & $12(100 \%)$ \\
\hline Improved HB & & $3(12 \%)$ & $2(14 \%)$ & $2(16.7 \%)$ \\
\hline \multicolumn{5}{|l|}{ Score } \\
\hline Worsened HB Score & & $0(0 \%)$ & $0(0 \%)$ & $0(0 \%)$ \\
\hline Improved GR Score & & $0(0 \%)$ & $0(0 \%)$ & $0(0 \%)$ \\
\hline Worsened GR Score & & $0(0 \%)$ & $0(0 \%)$ & $0(0 \%)$ \\
\hline
\end{tabular}

$\mathrm{n}=$ number, Avg = average, $\mathrm{cm}=$ centimeter, Gy = gray, VS = vestibular schwannoma, HB = House-Brackmann, GR = Gardner-

Robertson 
Table 2: Characteristics of patients lost to follow-up

\begin{tabular}{lllll}
\hline Sex & Age & Location & Presentation & Diameter of Tumor (cm) \\
\hline \hline M & 26 & Rt CPA + IAC & Ataxia, facial weakness + numb & 3.09 \\
M & 58 & Rt CPA + IAC & Hearing loss (NF-2) & 3.61 \\
F & 34 & Rt CPA & Facial numbness & 3.24 \\
\hline
\end{tabular}

$\mathrm{M}=$ male, $\mathrm{F}=$ female, $\mathrm{Rt}=$ right, $\mathrm{CPA}=$ cerebellar-pontine angle, $\mathrm{IAC}=$ internal acoustic canal, $\mathrm{cm}=$ centimeter, $\mathrm{NF}-2=$ neurofibromatosis type 2 .

analysis. Average follow-up period for the remaining patients was 34.5 months (range: 6-99 months). There were only 14 patients with greater than two years follow-up.

Of those lost to follow-up, one was followed clinically for 17 months and showed a worsened GR score from I to II as of last clinical contact. The other two patients were lost to follow-up after two months post GK at which point one patient had increased tinnitus with improvement in vertigo, ataxia, facial strength and numbness while the other reported an improvement in balance. Attempts to establish follow-up via multiple phone calls failed.

Twenty-one patients presented clinically with hearing deficits. Other presentations included: ataxia (18), vertigo (5), cranial nerve VI palsy (2), dysphagia (2), tinnitus (6), facial numbness (12), facial weakness (9), headache (6), diplopia (4), nystagmus (2), visual loss (1) and hydrocephalus (3). Clinical manifestations and their outcomes post-treatment can be seen in Table 3.

Overall, the average presenting GR grade of those patients with follow-up was 3.92 (range: 1-5). The number of patients with GR grades of I, II, III, IV, and V were 2, 5, 2, 0, and 16 respectively.

The average presenting $\mathrm{HB}$ grade was 2 (range: 1-6). The number of patients with presenting HB grades of I, II, III, IV, V, and VI were 13, 8, 0,1,1, and 2 respectively.

Average tumor maximum diameter was $3.28 \mathrm{~cm}$ (range: 3.0 $4.0 \mathrm{~cm}$ ). The anatomical distribution of tumors for the 25 patients were as follows: cerebellar-pontine angle (CPA) alone (1), CPA with internal acoustic canal (IAC) extension (1), CPA with brainstem compression (4), CPA with IAC extension, and brainstem compression (19).

\section{GK Treatment Parameters}

Treatment planning was achieved using MRI in all patients. Average 50\% isodose line dose was 12.5 Gy (range: 12-13 Gy). The average max dose was 25 Gy (range: 24-26 Gy). The average total volume covered (TVC) was $9.71 \mathrm{~cm}^{3}$ (range: 6.9 $10.6 \mathrm{~cm}^{3}$ ), with an average coverage of $97.2 \%$ (range: $91-100 \%$ ). Fifteen patients recorded a brainstem dose on average $12.3 \mathrm{~Gy}$ (range: 10.7-14.6 Gy).
Table 3: Presenting symptoms and response to Gamma knife treatment

\begin{tabular}{|c|c|c|}
\hline Symptom & $\begin{array}{l}\text { Number of } \\
\text { Patients }\end{array}$ & $\begin{array}{c}\text { Number of Patients } \\
\text { with Improvement (\%) }\end{array}$ \\
\hline Hearing Deficits & 21 & $0(0 \%)$ \\
\hline Ataxia & 18 & $10(55.6 \%)$ \\
\hline Vertigo & 5 & $5(100 \%)$ \\
\hline CN VI Palsy & 2 & $2(100 \%)$ \\
\hline Dysphagia & 2 & $1(50.0 \%)$ \\
\hline Tinnitus & 6 & $1(16.7 \%)$ \\
\hline Facial Numbness & 12 & $6(50.0 \%)$ \\
\hline Facial Weakness & 9 & $3(33.3 \%)$ \\
\hline Headache & 6 & $3(50.0 \%)$ \\
\hline Diplopia & 4 & $3(75.0 \%)$ \\
\hline Nystagmus & 2 & $0(0 \%)$ \\
\hline Visual Loss & 1 & $0(0 \%)$ \\
\hline Ventriculomegaly & 3 & $0(0 \%)$ \\
\hline
\end{tabular}

CN VI = cranial nerve VI. 


\section{Tumor Response to GK}

Tumor control was defined as either stable tumor size or decrease in size, as of last follow-up imaging. Twenty-three of 25 patients $(92.0 \%)$ displayed tumor control. Tumor size decreased in 14 of $25(56.0 \%)$ and remained stable in 9 of $25(36.0 \%)$. Average time until MRI change in size was 20.4 months.

In those patients with two years of follow-up group, 12 of 14 patients $(85.7 \%)$ displayed tumor control, of which ten $(71.4 \%)$ decreased in size and two (14.3\%) remained stable in size. Average time to decrease in tumor size post-GK was 24.8 months on MRI.

Two of the 25 patients $(14.3 \%)$ have tumor growth post -GK. One patient was a 36 -year-old male with a $4.0 \mathrm{~cm}$ left CPA VS, presenting with left hearing loss and ataxia. He received $12 \mathrm{~Gy}$ to the $50 \%$ isodose line with $96 \%$ coverage, yet at 21 months post-GK displayed tumor growth. He underwent surgical resection. The second patient was a 65 -year-old male with a 3.04 $\mathrm{cm}$ left CPA VS, presenting with left hearing loss, tinnitus and ataxia. He received 12 Gy to the $50 \%$ isodose line with $99 \%$ coverage. At 30 months post-GK this patient displayed increased tumor size, follow-up is continuing.

\section{Complications of Gamma Knife Radiosurgery}

Permanent complications following GK occurred in one patient displaying worsened hemifacial spasm. This patient was a 52-year-old female with a $3.3 \mathrm{~cm}$ CPA and IAC VS displaying brainstem compression. She received 13 Gy to the $50 \%$ isodose line and $98 \%$ tumor coverage. A brainstem dose of 12.7 Gy was recorded. Post-GK it was noted by the patient and her neurosurgeon that her pre-existing left hemifacial spasm was substantially worse. This has been persistent as of last follow-up.

Symptomatic hydrocephalus developed in four patients $(16.0 \%)$. One patient developed hydrocephalus two months post GK with subsequent right MCA infarct, unrelated to GK and contralateral to treatment side, at four months post treatment. This patient had other vascular risk factors pre-disposing them to a thrombo-embolic cause of the infarct. The second patient, with pre-treatment ventriculomegaly required VP shunt insertion following development of symptomatic hydrocephalus at 24 months post GK. The third patient had symptomatic progression of pre-existing ventriculomegaly requiring shunt insertion at 11 months post GK. Finally, the fourth patient, again with pretreatment ventriculomegaly, required shunt insertion for hydrocephalus at five months post GK. None of these patients displayed increased tumor size on post-GK MRI.

Minor transient symptoms were reported in 20 of $25(80 \%)$ of patients and included ataxia (8), numbness/tingling (9), facial weakness (2), neuralgia (2), dizziness (4), tinnitus (2), eyelid edema (10), headache (14), diplopia (2), nausea/vomiting (3), scalp numbness (1), ear pressure (1). All of these symptoms were absent as of last follow-up.

\section{Clinical Outcomes}

Among the 25 patients with follow-up, improvements in presenting symptoms were noted as follows: ataxia (10), dizziness or vertigo (5), improvement in facial strength (2), CN VI palsy (2), facial numbness (6), diplopia (3), dysphagia (1), headache (3), and tinnitus (1) This improvement in pre-GK clinical status did not correlate to decrease in tumor size, and was not expected nor implied to the patient pre-treatment. Presenting symptoms and improvement rates can be seen in Table 3.

Facial nerve preservation was good, with HB scores improving in three $(12 \%)$ by in person assessment by the treating surgeon. All three patients displayed a small decrease in tumor size as of last follow-up imaging. No patients demonstrated deterioration in pre-GK facial nerve function, with the exception of the patient with worsened hemifacial spasm. Similarly, no appreciable change, improvement or deterioration, was noted in GR score for any patient's post-GK. However, as mentioned in the Methods section, rarely did we have the objective audiology data available and subsequently relied on the audiologists/family doctor's letter indicating no change compared to pre-treatment.

\section{DISCUSSION}

Stereotactic radiosurgery for those VS smaller than $3 \mathrm{~cm}$ maximal diameter has been documented as an acceptable treatment alternative to microsurgical resection. Tumor control rates for these patients have been recorded in up to $94 \%{ }^{3}$ of cases. Similar long-term control has been described ${ }^{4}$. In addition, for those patients demonstrating tumor progression despite GK, repeat stereotactic radiosurgery can afford excellent tumor control ${ }^{10}$.

Facial nerve preservation rates, as determined by HB grading, have been reported at up to $98.2 \%$ with $\mathrm{GK}^{11,12}$ for small VS. Compared to microsurgery, the facial nerve preservation provided by GK has been shown to be superior ${ }^{1,2}$. Factors associated with facial nerve preservation post-GK are: a marginal dose of less than or equal to $13 \mathrm{~Gy}$, tumor volume less than $1.5 \mathrm{~cm}^{3}$, and age less than $60^{11}$.

Similarly, hearing preservation for small VS with GK has been documented at upwards of $85 \%$ for those patients presenting with GR grades of I to $\mathrm{III}^{13}$. Predictive factors for preservation of hearing are pre-GK GR grade of I, speech discrimination scores greater than $80 \%$, a pure tone average less than 20 decibels $(\mathrm{dB})$, age less than 60 years, and tumor volume less than $0.75 \mathrm{~cm}^{3}$. Also of significance are the radiation doses to the cochlea ${ }^{14}$, with a dose less than 4.2 Gy being predictive of hearing preservation ${ }^{15}$.

Finally, vestibular function post-GK in small VS has been difficult to predict. Changes in function of the vestibular system have been noted over time, with the most significant changes in the first six months post-GK. Literature documented rates of vestibular dysfunction post-GK have been shown in up to $57.4 \%$, with the majority resolving at six months follow-up ${ }^{16}$. Some develop late onset vestibular dysfunction (at six months or greater post-treatment). The postulated mechanism is related to post-GK edema and compression of the labyrinthine artery, impeding blood supply to the vestibular apparatus ${ }^{16}$.

Despite the promising results for VS with GK stereotactic radiosurgery, there are complications, defined as adverse radiation effects (ARE) associated with the treatment. Nonaudiofacial cranial neuropathy rates have been documented at $2.4 \%$, with the most common being trigeminal neuropathy ${ }^{17}$. Maintaining marginal doses less than $13 \mathrm{~Gy}$, especially in the area of the trigeminal nerve has been shown to reduce the risk of radiation induced trigeminal neuropathy ${ }^{12}$. Recently, trigeminal 
nerve dosing over $9 \mathrm{~Gy}$ is a possible predictor of post-GK dysfunction ${ }^{18}$. Besides non-audiofacial cranial neuropathy, hydrocephalus rates up to $6 \%$ have been reported in the literature post-GK, especially in those receiving marginal doses greater than $13 \mathrm{~Gy}^{17,18}$. The incidence of ARE post-GK for VS has been associated with treatment volume greater than $5 \mathrm{~cm}^{3}$.

Large vestibular schwannomas, defined as maximal diameter greater than $3 \mathrm{~cm}$, have a variety of treatment options available. Microsurgical resection and subtotal resection with goal for future stereotactic radiosurgery ${ }^{7}$ are currently the main treatment options. Recently, GK stereotactic radiosurgey alone as a primary treatment modality has been proposed for those VS of extra-canicular diameter 3 to $4 \mathrm{~cm}^{8,9}$. Stereotactic radiosurgery with GK alone has demonstrated an $89 \%$ tumor control rate at two years ${ }^{8}$ in the literature so far. Hearing, facial nerve, and trigeminal nerve preservation rates in these large VS treated with GK have been documented at $58 \%, 91 \%$, and $86 \%$ respectively 9 . Despite these promising early results, there is a lack of literature for this subset of VS in order to predict outcome and morbidity for GK as a primary treatment modality in these tumors.

Our series of 25 patients offers another look into the effects of GK as a primary treatment modality for those patients with VS of extra-canicular maximal diameter of 3-4 $\mathrm{cm}$. Our tumor control rate of $85.7 \%$ at two years is well within that described in the limited literature for large VS to date ${ }^{8,9}$. Only two patients (8\%) demonstrated tumor growth despite treatment. No particular treatment or patient demographic factors were predictive of their treatment failure.

We were able to avoid facial nerve and hearing deterioration in all patients, which is within literature limits described. However, our data for the objective GR grading postradiosurgery is limited in our charts, and as mentioned we had to rely on audiologist/family doctor communications of no change compared to pre-treatment. Our good facial nerve preservation rate is likely related to strict adherence to marginal dosing of 13 Gy or less, average age of 57 (less than 60), in addition to the majority of our patients (84\%) having a HB grade of I, II, or III pre-GK. Sixteen of $25(64 \%)$ patients had a GR grade of V prior to GK, indicating complete hearing loss and not subject to deterioration post-treatment. However, the good rates of hearing preservation in the remainder of the population treated, we believe are related strict marginal dose adherence.

Temporary vestibular dysfunction occurred in 7 of 25 patients (28\%), and was completely resolved as of last follow-up appointment. This was well within the rates previously defined in the literature for small VS, at up to $57.4 \%^{16}$.

Overall, our transient complication rate of $80 \%$ (20 of 25 patients) is higher than described in the literature for small VS, where ARE is quoted in up to $34 \%^{18}$. However, all of these complications were absent as of last follow-up. The majority of these transient complaints may be related to radiation irritation of surrounding structures. Anatomical distortion of neurologic structures secondary to the large size of these VS may predispose patients to develop radiation induced side-effects. One patient (4\%) had a permanent complication related to worsening hemifacial spasm after GK. This is still a treatment challenge as of last follow-up. Treatment parameters for this patient were in keeping with literature defined standards of marginal dosing of 13 Gy. This patient presented with HFS as her primary complaint, which persists and progresses further now after GK. Underlying vascular compression may remain the culprit cause. We acknowledge that the incidence of tumor related HFS is rare, with recent review indicating seven of 1642 cases $^{18}$. Partial surgical tumor debulking and microvascular decompression may be considered as future treatment. This pre-disposition to hemifacial spasm and a brainstem dose of 12.7 Gy recorded in this patient may be the cause of worsening hemifacial spasm post-GK. As a result, given the medically refractory nature of her worsened hemifacial spasm, we will strongly consider surgical decompression prior to stereotactic radiosurgery (SRS) in the future for patients with this specific presentation.

Shunt-dependent hydrocephalus developed in 4 of 25 patients (16\%) with follow-up. One patient displayed ventriculomegaly prior to GK, which worsened post-treatment requiring shunt placement for its symptomatic nature. This is higher than described in the literature for small $\mathrm{VS}^{19}$. This higher rate of hydrocephalus is likely related to the large size of these tumors, and possibly higher protein content of their cerebrospinal fluid.

Despite the large size of VS treated in our series, our results are promising. We believe our results for tumor control, hearing and facial nerve preservation, and a low rate of permanent complications are related to a few concepts. First, we maintained our marginal tumor dose at $13 \mathrm{~Gy}$ or less, which has been defined in the literature to provide optimal tumor control with low rates of $\mathrm{ARE}^{3,4}$. Second, maintenance of defined marginal doses and good pre-GK HB scores provided the opportunity for the facial nerve preservation we have achieved. Third, reducing the trigeminal nerve dose where possible to a goal of less than 13 Gy ${ }^{11,12}$, afforded us a low rate of temporary and permanent radiation induced trigmeninal neuropathy. Fourth, we strived to reduce the cochlear dose where possible ${ }^{15}$ in order to prevent post-GK hearing deterioration, though these dosing were not directly recorded, only taken into account during treatment planning. Finally, vigilance at maintaining tight treatment conformality, we believe, affords a high rate of tumor control and low permanent complication rate.

Despite our positive results, we do acknowledge that our series has limitations. First, the numbers are small with only 25 patients, making the results presented above difficult to generalize. Second, three patients were lost to follow-up. Thus, if all of these patients displayed treatment failure this could significantly impact our tumor control and complication rates. Third, our follow-up is short with only 14 of the 25 patients having two years or more. Knowing that the effects of GK can occur up to three years post treatment, the duration of follow-up we currently have is insufficient to make conclusions about longterm outcomes. Fourth, our low rate of hearing deterioration post-GK is largely related to the high number of patients with GR grade $\mathrm{V}$ hearing pre-GK. Thus, the rates of hearing preservation cannot necessarily be expected for all large VS. Sixth, given the retrospective nature of the study, the objective audiology data for post-radiosurgery assessment was lacking in our charts. As a result, we had to rely on audiologist/family physician communication of no change compared to preoperative measurements. One could argue we may be missing subtle changes in hearing without the objective numerical data to compare from both pre- to post-treatment. Finally, our complication rates are higher than those defined for small VS. 
This is likely related to the higher overall volume exposed to the treatment dose (as required for these large volume tumors), the significant anatomical distortion that one could postulate predisposes patients to ARE, and difficulty in reducing cochlear and brainstem dosing due to large treatment volumes. In addition, the incidence of ARE within our study may be understated secondary to the short follow-up. We do note, however, that the majority of our complications were transient in nature.

\section{Conclusions}

Gamma Knife stereotactic radiosurgery for the treatment of large VS of diameters 3 to $4 \mathrm{~cm}$ offers acceptable tumor control rates and good hearing and facial nerve preservation rates with a low permanent complication rate. Larger studies will need to be conducted in order to assess whether pre-existing marginal dose recommendations, and predictive factors of facial and hearing preservation and complications, as defined for small VS, apply for these larger tumors.

\section{REFERENCES}

1. Pollock BE, Driscoll CL, Foote RL, et al. Patient outcomes after vestibular schwannoma management: a prospective somparison of microsurgical resection and stereotacic radiosurgery. Neurosurgery. 2006;59:77-85.

2. Myrseth E, Moller P, Pedersen P, Lund-Johansen M. Vestibular schwannoma: surgery or Gamma Knife radiosurgery, A prospective, nonrandomized study. Neurosurgery. 2009;64(4): 654-63.

3. Timmer FCA, Hanssens PEJ, van Haren AE, et al. Follow-up after Gamma Knife radiosurgery for vestibular schwannomas: volumetric and axial control rates. Laryngoscope. 2011;121: 1359-66.

4. Chopra R, Kondziolka D, Nironjan A, Lunsford LD, Flickinger JC. Long-term follow-up of acoustic schwannoma radiosurgery with marginal tumor doses of 12 to $13 \mathrm{~Gy}$. Int J Radiation Oncology Biol Phys. 2007:68(3):845-51.

5. Murphy ES, Barnett GH, Vogelbaum MA, et al. Long-term outcomes of Gamma Knife radiosurgery in patients with vestibular schwannomas. J Neurosurg. 2011;114:432-40.

6. Roos DE, Potter AE, Brophy BP. Stereotactic radiosurgery for acoustic neuromas: what happens long term. Int J Radiation Oncology Biol Phys. 2012;82(4):1352-5.
7. Van de Langenberg R, Hanssens PEJ, van Overbeeke JJ, et al. Management of large vestibular schwannoma. Part 1. planning subtotal resection followed by Gamma Knife surgery: radiological and clinical aspects. J Neurosurg. 2011;115:875-84.

8. Yang H, Kano H, Awan NR, et al. Gamma Knife radiosurgery for larger-volume vestibular schwannomas. J Neurosurg. 2011;114: 801-7.

9. Van de Langenberg R, Hanssens PEJ, Verheul JB, et al. Management of large vestibular schwannoma. part II. primary Gamma Knife surgery: radiological and clinical aspects. J Neurosurg. 2011; 115:885-93.

10. Kano H, Kondziolka D, Niranjan A, et al. Repeat stereotactic radiosurgery for acoustic neuromas. Int $\mathrm{J}$ Radiation Oncology Biol Phys. 2010; 76(2):520-7.

11. Yang I, Sughrue ME, Han SJ, et al. Facial nerve preservation after vestibular schwannoma Gamma Knife radiosurgery. J Neurooncol. 2009;93:41-8.

12. Lobato-Polo J, Kondziolka D, Zorro O, Kano H, Flickinger JC, Lunsford LD. Gamma Knife radiosurgery in younger patients with vestibular schwannomas. Neurosurgery. 2009;65(2): 294-301.

13. Niranjan A, Mathieu D, Flickinger JC, Kondziolka D, Lunsford LD. Hearing preservation after intracanalicular vestibular schwannoma radiosurgery. Neurosurgery. 2008;63(6):1054-64.

14. Kano H, Kondziolka D, Khan A, Flickinger JC, Lunsford LD. Predictors of hearing preservation after stereotactic radiosurgery for acoustic neuroma. J Neurosurg. 2009; 111:863-73.

15. Timmer FCA, Hanssens PEJ, van Haren AEP, et al. Gamma Knife radiosurgery of vestibular schwannomas: results of hearing preservation in relation to the cochlear radiation dose. Laryngoscope. 2009;119:1076-81.

16. Wackym PA, Hannley MT, Runge-Samuelson CL, Jensen J, Zhu YR. Gamma Knife surgery of vestibular schwannomas: longitudinal changes in vestibular function and measurement of the dissiness handicap inventory. J Neurosurg. 2008;109:137-43.

17. Sughrue ME, Yang I, Han SJ, et al. Non-audiofacial morbidity after Gamma Knife surgery for vestibular schwannoma. Neurosurg Focus. 2009;26(4):E4

18. Han IB, Chang JH, Chang JW, et al. Unusual causes and presentations of hemifacial spasms. Neurosurgery. 2009;65(1): 130-7.

19. Hayhurst C, Monsalves E, Bernstein M, et al. Predicting nonauditory adverse radiation effects following radiosurgery for vestibular schwannoma: a volume and dosimetric analysis. Int $\mathbf{J}$ Radiation Oncology Biol Phys. 2012;82(5):2041-6. 